\title{
Tear calcium levels and contact lens wear
}

\author{
A. F. WINDER, M. RUBEN, AND G. A. K. SHERAIDAH \\ From the Department of Pathology, Institut: of Ophthalmology, and Department of Contact Lens and \\ Prosthetics, Moorfields Eye Hospital, London
}

SUMMARY Hydrophilic contact lens spoliation can be associated with the deposition of calcium salts. The relationship between the concentration of calcium in tear fluid and contact lens use has been studied in healthy volunteers and in patients using various forms of hard and soft contact lenses, tear samples being collected with Schirmer strips and with lenses in situ. Methods are suggested for correcting data on calcium per strip to concentration when this cannot be directly determined. Further data were obtained by direct extraction of freshly removed soft contact lenses. The collected results indicate some variation in tear calcium concentrations, but values are unrelated to use of contact lens and type of lens fitted, and to the rates of tear flow with our procedures for collection. Local calcium concentrations are unlikely therefore to be a significant primary factor in soft contact lens spoliation, but the enlargement of the tear pool associated with the use of a soft contact lens does greatly increase the amount of calcium present, and this may be a factor in secondary deposition.

Deposition of calcium salts can be a significant factor in the spoliation of hydrophilic contact lenses (Ruben et al., 1975). It is likely that lachrymal fluid is at least a major source of the calcium deposited, and studies of tear composition in association with contact lens wear are therefore of interest. Uotila et al. (1972) reported that normal values for tear calcium concentration displayed a narrow range and were unrelated to serum levels, and found similar results in a small series of patients wearing contact lenses, including 2 wearing soft lenses. Flow rate and thus, presumably, the degree of tear stimulation associated with lens use and sample collection may also be involved (Botelho et al., 1973).

We have determined calcium and, in part, sodium and potassium for lachrymal fluid obtained from patients with various forms of hard and particularly soft contact lenses in situ, from patients with corneal lesions but not using contact lenses, and from apparently normal individuals. We obtained these data to give an idea of the range of calcium concentrations found in the lachrymal sac under various conditions, and to see if there could therefore be a simple relationship between local calcium concentration and deposition processes in the hydrophilic contact lens and possibly in the cornea also.

Address for reprints: Dr A. F. Winder, Department of Pathology, Institute of Ophthalmology, Judd Street, London WC1 9QS

\section{Methods and patients}

This study was on 133 eyes in 74 patients -37 male, 37 female-with an age range of 19 to 67 years. The eyes were in the following groups.

Contact lens use.-Tear samples were obtained by Schirmer strip collection without accessory stimulation from 22 patients, 32 eyes, with various types of lens in situ, as follows: hydrophilic lenses, 8 patients, 10 eyes; corneal hard lenses, 9 patients, 13 eyes; scleral hard lenses, 5 patients, 9 eyes. Hydrophilic lenses were directly removed for analysis from a further group of 33 patients (57 eyes) who were experienced and symptom-free users. The lenses had been worn for several hours before removal.

Without lenses.-Schirmer strip samples were taken from the unfitted eye in 5 cases in which use of contact lens was monocular. Sixteen samples were taken from 30 eyes-mostly pooling left and right samples-from apparently normal individuals and 13 samples from 6 patients with corneal disease not involving use of a contact lens-old herpes, keratoconus, corneal opacification, squint not improved by use of contact lens, corneal irritation (repeat samples on different occasions), and conjunctival accretions.

SAMPLE HANDLING

Most of the Schirmer strip samples from patients were taken during visits to the hospital contact lens 
clinic and sent to the laboratory in special acidwashed containers or in the polyethylene envelope in which the strips are supplied. Control studies confirmed that these methods were satisfactory, but care is necessary here: some trial specimens were delivered to the laboratory wrapped in hospital clinical notepaper, which was found to be a significant source of calcium. Although the importance of handling the Schirmer strips with forceps only was emphasised, the handling conditions away from the laboratory were uncertain. Strips were therefore bisected, and the possibly handled distal portion was rejected before analysis.

Samples for 28 normal eyes-14 pooled left and right eye samples-and 4 samples collected at 2 visits from 1 patient complaining of uniocular corneal irritation but in whom no abnormality could be found were taken at the laboratory under supervision. Forceps were used to avoid handling of the strips, and where a good flow was achieved whole strips were rapidly transformed to closed acid-washed containers, weighed, lyophilised, and reweighed to determine volume of tears collected.

Gel contact lenses for analysis were removed by the patient after careful hand washing and conveyed to the laboratory in closed acid-washed containers. Some patients could visit the laboratory for lens removal, and in 19 patients (33 eyes) very rapid removal and transfer to containers was achieved. These samples were lyophilised to determine the fluid volume of the lens before analysis. Control in-vitro studies had shown that lens salt content was effectively in equilibrium with the incubation medium after 6 hours. All gel lenses in this group of patients had been worn for at least 6 , and in most cases more than 8 hours before removal of the lens for analysis. Removed lenses were lyophilised, reweighed, extracted, washed, and returned to the patient within 24 hours. Of the 57 lenses examined only one complaint of alleged change in the properties of the lens was received.

\section{ELECTROLYTE ANALYSIS}

Salts were extracted by soaking the strips for several hours with intermittent agitation in aqueous $0 \cdot 125 \% / 0 \cdot 125 \%$ lanthanum chloride/formic acid. Calcium, sodium, and in some cases potassium were determined on these solutions by aspiration into the burner of a Shandon Southern A3400 atomic absorption spectrophotometer. All results were determined at least in duplicate, the apparatus being continuously calibrated with a range of blank and standard solutions.

CALCULATION OF RESULTS

The volume of samples collected in the laboratory, either with lenses or with Schirmer strips, was determined by lyophilisation and reweighing. This volume approach in the strip series was restricted to rapid-flow cases, where loss by evaporation from the strips during collection would be proportionally slight and no correction was applied. This factor does not arise in the lens series, but selective binding of water and/or salts to the lens matrix, together with the presence of deposited material, may result in the composition of lens extracts being unrepresentative of the adjacent tear film. All lenses were undergoing regular cleaning and were operating satisfactorily at the time of analysis, and a deposit contribution was unlikely to be significant. The other artefacts depend on lens type, and our in vitro lens incubation studies indicate that with calcium the errors involved are not gross. Thus with Plano-T lenses calcium artefacts are of no practical significance, but high water content Sauflon lenses-for example, Sauflon 85-may contain about twice the amount of calcium predicted from the contained fluid volume. Even so, the amount of calcium present in both in-vivo and in-vitro studies in no case exceeded $12 \mu \mathrm{g}$, whereas values of up to $250 \mu \mathrm{g}$ of calcium were recorded for a series of spoilt gel lenses (Ruben et al., 1975).

A direct determination of tear volume was not possible on Schirmer strip samples forwarded from the hospital, for which the amount of calcium present per bisected strip was determined. We have attempted to estimate tear calcium concentration from these data. Pilot experiments indicated that, on the assumption the wet edge passed the midpoint, then strip bisection corresponded to a collected volume of approximately $10 \mu \mathrm{l}$, with, however, some variation depending on the rate of tear flow on to the strip. Calcium concentration may thus be estimated. For example, $0 \cdot 2 \mu \mathrm{g}$ per strip of $10 \mu \mathrm{l}$ of tears is equivalent to $2 \mathrm{mg}$ of calcium per $100 \mathrm{ml}$. A second approach to this correction derives from our determination of sodium per bisected strip. Our further studies of normal tears collected similarly but with direct measurement of tear volume, and thus sodium concentration, indicated an average value for tear sodium close to $120 \mathrm{mmol} / 1$ in good agreement with previous authors (Lowther et al., 1970). Given the sodium content per bisected strip, we can estimate the collected tear volume and thus the calcium concentration. We have applied and compared these corrections widely, and the sodiumbased procedure gives rather more compact normal ranges, with some reduction in mean calcium, concentrations, principally because occasional very high values obtained by the volumetric procedure are much reduced; most results-that is, the lower values-are little changed. We prefer the sodium- 
based procedure, because small samples can still provide useful results. Derived data as shown in Figs. 1 and 2 are produced by this procedure. Possibly these procedures do not hold closely for the individual case, but the error is most unlikely to exceed $\pm 15 \%$, and we believe that a useful overall view of the pattern of results is obtained.

In our experience the direct collection of useful volumes of tears by capillary tube methods without lachrymatory agents can involve an unacceptable degree of mechanical stimulation, and the alternative methods used in this study remain important in spite of their difficulties.

\section{Results}

All numerical data are summarised in Figs. 1 to 4 .

Tear calcium in association with contact lens use

(a) Schirmer strip collection. Values for calcium per bisected strip ranged from 0.08 to $1.7 \mu \mathrm{g}$, with most values below $0.5 \mu \mathrm{g}$. Volume correction on the sodium-based procedure indicated a range of tear calcium concentration from 0 to $2 \cdot 2 \mathrm{mmol} / 1$, with most values below $1.25 \mathrm{mmol} / 1 \quad(5 \mathrm{mg} / 100 \mathrm{ml})$. Results for corneal, scleral, and gel lenses are recorded separately in Figs. 1 and 2.

(b) Soft lens extraction. The calcium content of the various hydrophilic lens types extracted ranged from 0.3 to $12 \mu \mathrm{g}$, with most values below $3 \mu \mathrm{g}$ per lens. The enclosed tear volume was recorded for 34 of these lenses (range 16.8 to $35.4 \mu \mathrm{l}$ ), suggesting a range for calcium concentration from 0.35 to $4.0 \mathrm{mmol} / \mathrm{l}$, again in a skew distribution, with most values below $1.0 \mathrm{mmol} / \mathrm{l}$. These results are shown in Figs. 3 and 4, together with the normal range for tear calcium reported by Uotila et al. (1972).

Tear calcium from normal and pathological eyes without contact lens use

(a) Schirmer strip collection, estimating tear volume (the 'no lens' group). Values for calcium per bisected strip in this mixed group ranged from 0 to $1.7 \mu \mathrm{g}$, with most values below $1 \mu \mathrm{g}$, equivalent to a concentration around $1.25 \mathrm{mmol} / \mathrm{l}$ calculated from the sodium content as above.

(b) Schirmer strip collection, with direct measurement of collected tear volume (the 'normal' group). Values for calcium concentration ranged from 0.33 to $2.4 \mathrm{mmol} / \mathrm{l}$, with most values below $1 \mathrm{mmol} / \mathrm{l}$.

Results for these 2 groups are shown in Figs. 1 and 2 without differentiation of the pathological eye results, which did not in general differ from the pattern in each group. Two minor exceptions were a case of perforated cornea to which a soft lens was applied and later found to contain $11.8 \mu \mathrm{g}$ of

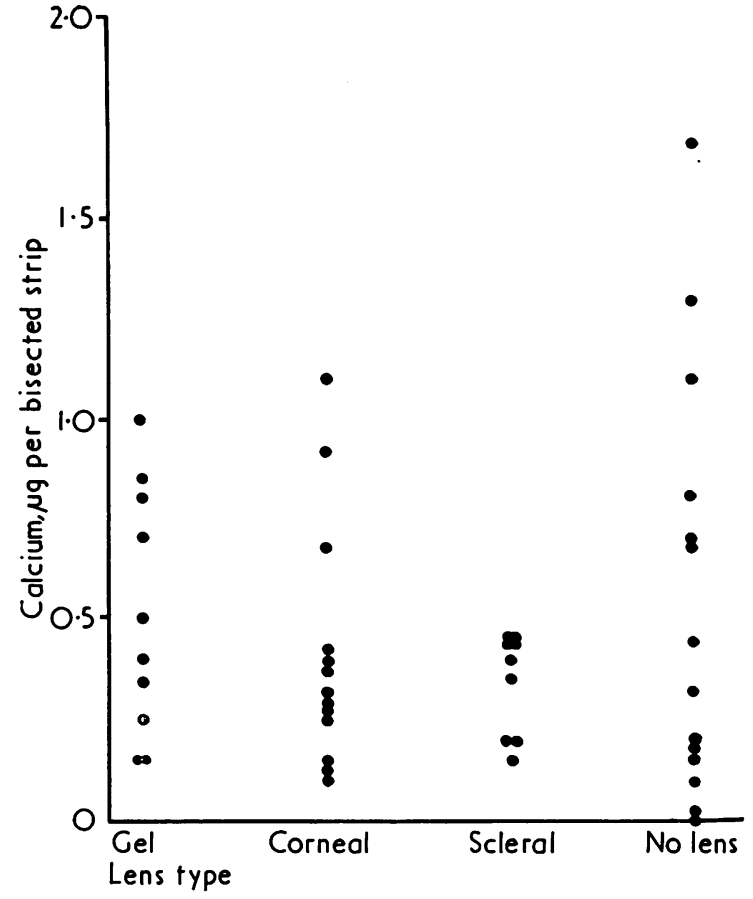

Fig. 1 Amount of calcium per bisected strip; fluid collected from eyes with and without contact lenses

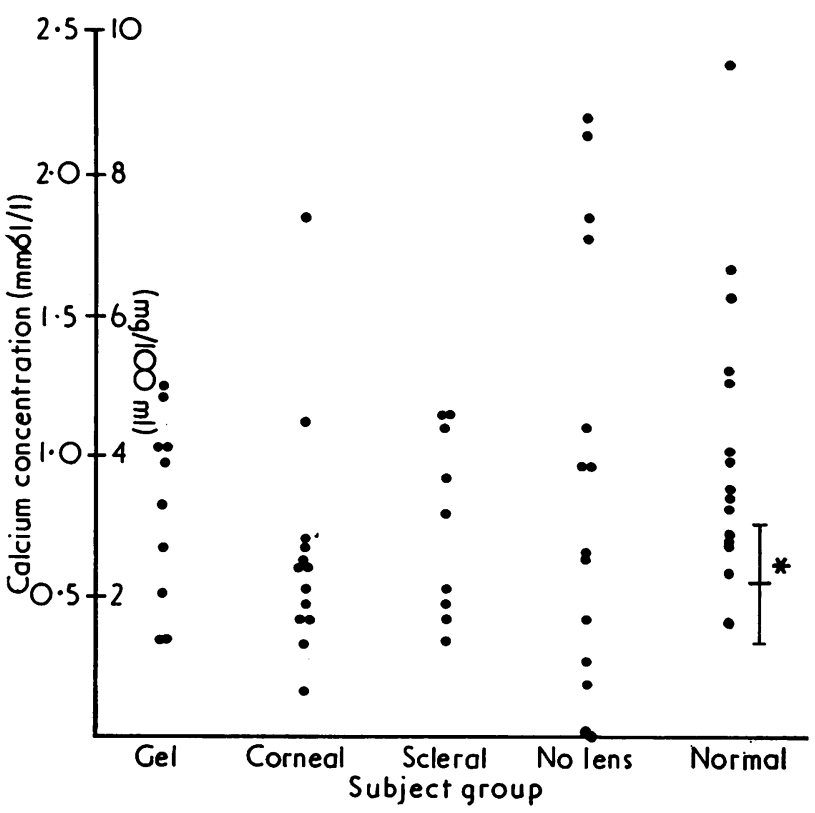

Fig. 2 Tear calcium concentration for various groups of subjects, with and without different forms of contact lens. The normal range data of Uotila et al. (1972) is also shown* 


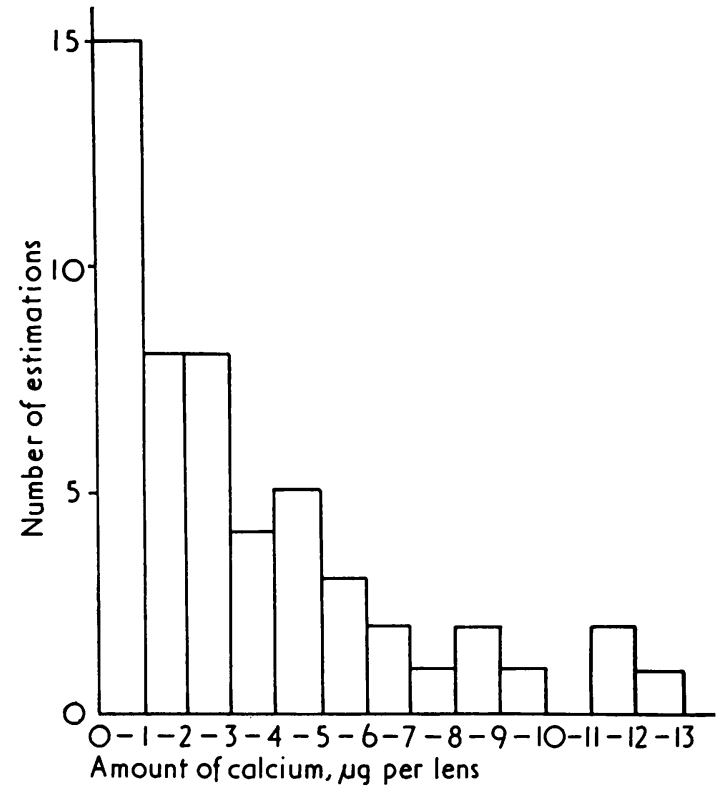

Fig. 3 Amount of calcium extracted from soft contact lenses-1

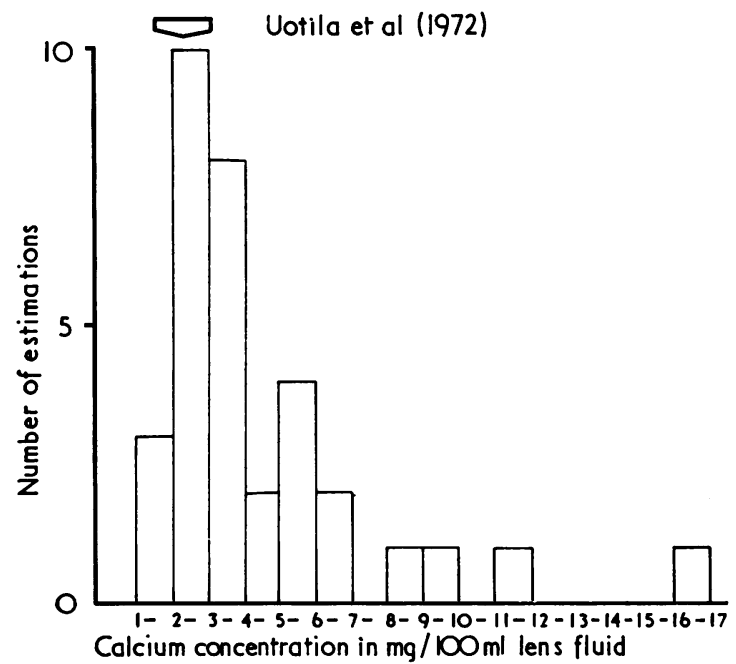

Fig. 4 Amount of calcium extracted from soft contact lenses-2

calcium, and the woman with uniocular conjunctival irritation, for whom a value of $2.47 \mathrm{mmol} / 1$ was recorded on one occasion for the allegedly normal eye.

\section{Relationship between flow rate and tear calcium concentration}

Flow rate in 15 of the normal volunteer subjects was assessed on a 1-to-4-plus scale, using Schirmer strip collection and direct concentration analysis. Graphically a correlation between flow rate and calcium concentration was not obvious, and the calculated correlation coefficient of +0.193 supported this view.

\section{Discussion}

How might calcium levels in tear fluid be related to contact lens use? Tear fluid is of diverse origin and there are defined lachrymal gland and epithelial components. Tear potassium levels are certainly affected by contact lens use and type of lens fitted (Winder and Ruben, 1977), and this may reflect changes in epithelial turnover in the cornea and/or conjunctiva. Botelho et al. (1973) showed that tear calcium levels in man are much higher at basal rates of flow, and from animal work it may be inferred that this effect applies to both lachrymal gland fluid and to other components of the tear film (Horowitz et al., 1976). The presence of calcium in lens cleaning fluids was shown to be associated with opacification (Lowther and Hilbert, 1975), and thus local concentrations - that is, tear fluid levels-may contribute.

The principal objective of this study was to assess the range of calcium availability in tear fluid, with and without contact lens use, and we feel that our diverse approach with some reliance on indirect and less precise methods gives a useful overall picture. In fact a relationship between tear calcium levels and contact lens use has not been established, and the preliminary results of Uotila et al. (1972) are confirmed, although we find a rather wider range of variation than that previously reported. Tear collection by capillary tube or Schirmer strip involves a degree of stimulation, and this effect could be sufficient to reduce calcium concentrations to a plateau level, thus obscuring a contact lens influence. However, we obtained similar results when tears were collected from patients experienced in wearing contact lenses by using Schirmer strips with lenses in situ, or by direct soft lens analysis. It seems therefore that this artefact does not arise, and this agreement also suggests that at least for the various hydrophilic lens types involved in this study the presence of the lens affords sufficient stimulus to maintain tear fluid calcium levels at or near those of extracellular fluid. We have recorded the occasional higher value for tear calcium concentration, particularly after indirect methods of collection, but the overall pattern indicates that, in the conditions of flow rate involved, elevated tear calcium levels in man are at most very uncommon and may not arise. High values were not confirmed when a repeat determination was possible, and the 
presence of deposit may be contributory. The measurement of tear calcium levels in association with contact lens spoilation is, however, always worth considering, as patients with elevated levels in this special group may yet be recognised. The suggestion of Uotila et al. (1972) that this effect might arise in sarcoid has apparently not been pursued.

Since the concentration of calcium in tears is unlikely to be a significant influence in spoliation, what other factors could be involved? Calcium deposition may in many, and perhaps all, cases be a secondary process, and mechanical damage and/or lipid deposition may be significant primary events (Doughman et al., 1975). It is worth noting that, however the calcium involved in lens deposits is laid down, significant amounts are found in tears and in contact lens fluid, and that the tear pool volume may exceed $70 \mu \mathrm{l}$ in the presence of a gel lens of high water content, representing a considerable increase in the amount if not the concentration of calcium present.
We thank the Moorfields Eye Hospitals Research Committee for a grant under the scheme for locally organised clinical research, and the staff of the contact lens department for assistance at all times.

\section{References}

Botelho, S. Y., Goldstein, A. M., and Rosenlund, M. L. (1973). Journal of Pediatrics, 83, 601.

Doughman, D. J., Mobilia, E., Drago, D., Havener, V., and Gavin, M. (1975). Annals of Ophthalmology, 7, 345.

Horowitz, G., Lammers, J. W., Sussman, S., and Botelho, S. Y. (1976). Investigative Ophthalmology, 15, 994.

Lowther, G. E., and Hilbert, J. A. (1975). American Journal of Optometry and Physiological Optics, 52, 687.

Lowther, G. E., Miller, R. B., and Hill, R. M. (1970). American Journal of Optometry and Physiological Optics, 47, 266.

Ruben, M., Tripathi, R. C., and Winder, A. F. (1975). British Journal of Ophthalmology, 59, 141.

Uotila, M. H., Soble, R. E., and Savory, J. (1972). Investigative Ophthalmology, 11, 258.

Winder, A. F., and Ruben, M. (1977). Transactions of Ophthalmological Societies of the United Kingdom (In press). 